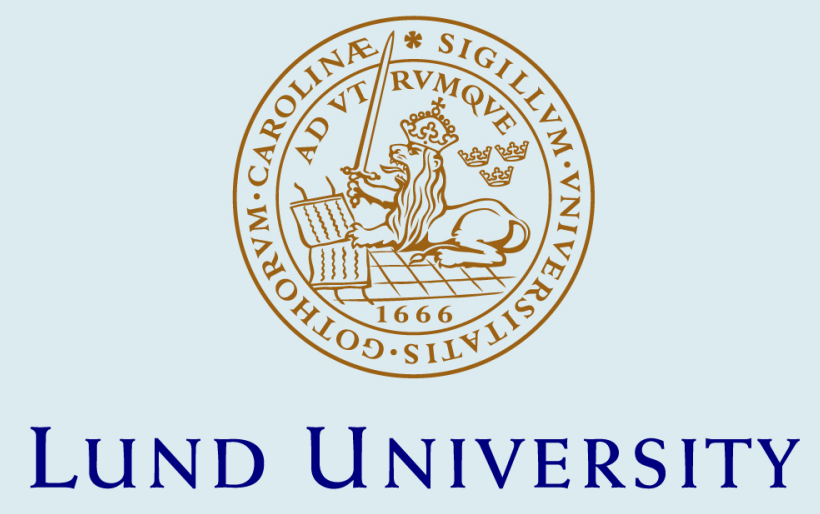

\title{
Distance bounds for periodically time-varying and tail-biting LDPC convolutional codes
}

Truhachev, Dmitri; Zigangirov, Kamil; Costello, Daniel

Published in:

IEEE Transactions on Information Theory

DOI:

10.1109/TIT.2010.2053873

2010

Link to publication

Citation for published version (APA):

Truhachev, D., Zigangirov, K., \& Costello, D. (2010). Distance bounds for periodically time-varying and tail-biting LDPC convolutional codes. IEEE Transactions on Information Theory, 56(9), 4301-4308.

https://doi.org/10.1109/TIT.2010.2053873

Total number of authors:

3

\section{General rights}

Unless other specific re-use rights are stated the following general rights apply:

Copyright and moral rights for the publications made accessible in the public portal are retained by the authors

and/or other copyright owners and it is a condition of accessing publications that users recognise and abide by the legal requirements associated with these rights.

- Users may download and print one copy of any publication from the public portal for the purpose of private study or research.

- You may not further distribute the material or use it for any profit-making activity or commercial gain

- You may freely distribute the URL identifying the publication in the public portal

Read more about Creative commons licenses: https://creativecommons.org/licenses/

Take down policy

If you believe that this document breaches copyright please contact us providing details, and we will remove access to the work immediately and investigate your claim. 


\title{
Distance Bounds for Periodically Time-Varying and Tail-Biting LDPC Convolutional Codes
}

\author{
Dmitri Truhachev, Member, IEEE, Kamil Sh. Zigangirov, Fellow, IEEE, and \\ Daniel J. Costello, Jr., Fellow, IEEE
}

\begin{abstract}
Existence type lower bounds on the free distance of periodically time-varying LDPC convolutional codes and on the minimum distance of tail-biting LDPC convolutional codes are derived. It is demonstrated that the bound on free distance of periodically time-varying LDPC convolutional codes approaches the bound on free distance of general (non-periodic) time-varying LDPC convolutional codes derived in [1] as the period increases. The proof of the bound is based on lower bounding the minimum distance of corresponding tail-biting LDPC convolutional codes, which is of interest in its own right.
\end{abstract}

\section{Index Terms}

Free distance, minimum distance, lower bounds, low-density parity-check (LDPC) codes, LDPC convolutional codes, tail-biting LDPC convolutional codes.

\section{INTRODUCTION}

LDPC block codes were invented by Gallager [2] in the 1960s. The construction of the corresponding convolutional counterparts, LDPC convolutional codes (LDPCCCs), was first

This work was supported in part by NSF Grants CCR-02-05310 and CCF05-15012, NASA Grant NNX07AK53G, and the Alberta Ingenuity Fund.

D. Truhachev is with the Electrical and Computer Engineering Research Faculty (ECERF), University of Alberta, Edmonton, Alberta, Canada T6G 2V4

K. Sh. Zigangirov is with the Institute for Problems of Information Transmission, Moscow, Russia, and the Department of Electrical Engineering, University of Notre Dame, Notre Dame, IN 46556, USA.

Daniel J. Costello, Jr., is with the Department of Electrical Engineering, University of Notre Dame, Notre Dame, IN 46556, USA. 
presented $^{1}$ in [3]. While minimum distance bounds for block LDPCs were derived in Gallager's original work [2], the first analytical lower bound on the free distance of LDPCCCs was only derived recently [1]. The proof presented in [1] holds for an ensemble of general (non-periodic) time-varying LDPCCCs and must employ a special expurgation technique to compensate for the non-periodic structure of the ensemble.

In this paper, we derive an existence type lower bound on the free distance of periodically timevarying LDPCCCs. We show that, as the period increases, the new bound approaches the bound on free distance of non-periodic LDPCCCs derived in [1]. The proof presented for the new bound is based on considering the minimum distance of tail-biting LDPCCCs (TB-LDPCCCs) [8]. In particular, we lower bound the minimum distance of TB-LDPCCCs constructed from an ensemble of periodically time-varying LDPCCCs and use this to lower bound the free distance of the original ensemble.

Tail-biting was introduced by Solomon and van Tilborg [9] and independently by Ma and Wolf [10] as a method of terminating a convolutional code without the rate loss caused by standard termination. The resulting tail-biting codes have a dual nature, i.e., they simultaneously have the properties of both block and convolutional codes. As a consequence, their minimum distance depends both on the block length of the tail-biting code and the constraint length of the convolutional code.

The minimum distance of conventional (non-LDPC) tail-biting codes equals the minimum of two related distance measures, $d_{\text {intra }}$ and $d_{\text {inter }}$ [11]. The intra minimum distance $d_{\text {intra }}$ reflects the convolutional code properties of the tail-biting code and is lower bounded by the Costello bound [6] on the free distance of convolutional codes. The inter minimum distance $d_{\text {inter }}$ reflects the block code properties of the tail-biting code and is lower bounded by the Varshamov-Gilbert bound [4], [5] on the minimum distance of block codes. Analogous to conventional tail-biting convolutional codes, the minimum distance of TB-LDPCCCs is lower bounded by the minimum of $d_{\text {intra }}$ and $d_{\text {inter }}$, where $d_{\text {intra }}$ is lower bounded by the bound on free distance of LDPCCCs derived in [1] and $d_{\text {inter }}$ is lower bounded by Gallager's bound on minimum distance of LDPC block codes [2].

The paper is organized as follows. Section II presents the definition of the LDPCCC code

${ }^{1}$ The basic idea of LDPCCCs was first described in Tanner's patent application [7]. 
ensemble considered. Section III is devoted to lower bounding the minimum distance of TBLDPCCCs, and a lower bound on the free distance of periodically time-varying LDPCCCs is proved in Section IV. Numerical results are given in Section V, and Section VI concludes the paper.

\section{An LDPC Convolutional Code Ensemble}

In [3], a rate $R=b / c$ binary convolutional code was defined as the set of sequences $\boldsymbol{v}_{[0, \infty]}=\left(\boldsymbol{v}_{0}, \boldsymbol{v}_{1}, \ldots\right), \boldsymbol{v}_{t} \in \mathbb{F}_{2}^{c}$, satisfying the equality $\boldsymbol{v}_{[0, \infty]} \boldsymbol{H}_{[0, \infty]}^{\mathrm{T}}=\mathbf{0}$, where the semi-infinite syndrome former (transposed parity-check) matrix $\boldsymbol{H}_{[0, \infty]}^{\mathrm{T}}$ is given by

$$
\boldsymbol{H}_{[0, \infty]}^{\mathrm{T}}=\left(\begin{array}{ccccc}
\boldsymbol{H}_{0}^{\mathrm{T}}(0) & \ldots & \boldsymbol{H}_{m_{\mathrm{s}}}^{\mathrm{T}}\left(m_{\mathrm{s}}\right) & & \\
& \ddots & & \ddots & \\
& & \boldsymbol{H}_{0}^{\mathrm{T}}(t) & \ldots & \boldsymbol{H}_{m_{\mathrm{s}}}^{\mathrm{T}}\left(t+m_{\mathrm{s}}\right) \\
& & \ddots & & \ddots
\end{array}\right)
$$

and each entry $\boldsymbol{H}_{i}^{\mathrm{T}}(t+i)$ is a $c \times(c-b)$ binary matrix. To satisfy an easy encoding property (see [3], [13]), the matrices $\boldsymbol{H}_{0}^{\mathrm{T}}(t)$ must have full rank for all time instants $t$, and hence we assume that the last $(c-b)$ rows of $\boldsymbol{H}_{0}^{\mathrm{T}}(t)$ are linearly independent for all $t$. Then the first $b$ symbols of $\boldsymbol{v}_{t}$ at each time instant $t$ are information symbols and the last $(c-b)$ symbols are parity symbols. The largest $i$ such that $\boldsymbol{H}_{i}^{\mathrm{T}}(t+i)$ is a non-zero matrix for some $t$ is called the syndrome former memory $m_{\mathrm{s}}$. A $(J, K)$ regular $L D P C C C$ is defined by a syndrome former that contains exactly $J$ ones in each row and $K$ ones in each column (starting from the $\left((c-b) m_{\mathrm{s}}+1\right)$ th column).

Now we define a special sub-class of $(J, K)$ regular LDPCCCs, where the component submatrices $\boldsymbol{H}_{i}^{\mathrm{T}}(t)$ are composed of $M \times M$ binary permutation matrices ${ }^{2}$. Let $a=\operatorname{gcd}(J, K)$ denote the greatest common divisor of $J$ and $K$. Then there exist positive integers $J^{\prime}$ and $K^{\prime}$ such that $J=a J^{\prime}$ and $K=a K^{\prime}$ and $\operatorname{gcd}\left(J^{\prime}, K^{\prime}\right)=1$. For $i=0,1, \ldots, a-1$, the $K^{\prime} M \times J^{\prime} M$

\footnotetext{
${ }^{2}$ This subclass was considered in [1] in order to prove a lower bound on $d_{\text {free }}$ for non-periodic LDPCCCs.
} 
sub-matrices $\boldsymbol{H}_{i}^{\mathrm{T}}(t+i)$ of the syndrome former are

$$
\boldsymbol{H}_{i}^{\mathrm{T}}(t+i)=\left(\begin{array}{cccc}
\boldsymbol{P}_{i}^{(0,0)}(t+i) & \boldsymbol{P}_{i}^{(0,1)}(t+i) & \ldots & \boldsymbol{P}_{i}^{\left(0, J^{\prime}-1\right)}(t+i) \\
\boldsymbol{P}_{i}^{(1,0)}(t+i) & \boldsymbol{P}_{i}^{(1,1)}(t+i) & \ldots & \boldsymbol{P}_{i}^{\left(1, J^{\prime}-1\right)}(t+i) \\
\ldots & \ldots & \ldots & \ldots \\
\boldsymbol{P}_{i}^{\left(K^{\prime}-1,0\right)}(t+i) & \boldsymbol{P}_{i}^{\left(K^{\prime}-1,1\right)}(t+i) & \ldots & \boldsymbol{P}_{i}^{\left(K^{\prime}-1, J^{\prime}-1\right)}(t+i)
\end{array}\right),
$$

where each $\boldsymbol{P}_{i}^{(k, j)}(t+i), k=0,1, \ldots, K^{\prime}-1, j=0,1, \ldots, J^{\prime}-1$, is an $M \times M$ permutation matrix. All other entries of the syndrome former are $K^{\prime} M \times J^{\prime} M$ zero matrices. We assume that the matrix $\boldsymbol{H}_{[0, \infty]}^{\mathrm{T}}$ is periodically time-varying with period $T$, i.e.,

$$
\boldsymbol{H}_{i}^{\mathrm{T}}(t+i)=\boldsymbol{H}_{i}^{\mathrm{T}}(t+i+T) \quad \forall i, t .
$$

In this case, a code is characterized by a section $\boldsymbol{H}_{[0, T-1]}^{\mathrm{T}}$ of the semi-infinite syndrome former $\boldsymbol{H}_{[0, \infty]}^{\mathrm{T}}$.

A syndrome former $\boldsymbol{H}_{[0, T-1]}^{\mathrm{T}}$ for a periodically time-varying $(3,6)$ regular LDPCCC is shown in Fig. 1. In this case, the code construction parameters are $K=6, J=3, a=3, c=2 M$, and $b=M$. Each matrix $\boldsymbol{H}_{i}^{\mathrm{T}}(t+i)$ consists of two $M \times M$ permutation matrices, i.e.,

$$
\boldsymbol{H}_{i}^{\mathrm{T}}(t+i)=\left(\begin{array}{c}
\boldsymbol{P}_{i}^{(0)}(t+i) \\
\boldsymbol{P}_{i}^{(1)}(t+i)
\end{array}\right),
$$

where (4) has full rank equal to $M$. Therefore the code rate is $M / 2 M$. Note that by permuting rows of the syndrome former, an equivalent rate $1 / 2(3,6)$ regular LDPCCC with syndrome former memory at most $3 M-1$ can be obtained (see [1]).

Now suppose that the $M \times M$ permutation matrices comprising the sub-matrices (2) of the syndrome former $\boldsymbol{H}_{[0, T-1]}^{\mathrm{T}}$ are chosen independently and such that each of the $M$ ! possible permutation matrices is equally likely. Then we obtain a random ensemble of $(J, K)$ regular T-periodic LDPCCCs, which we designate $\mathcal{C}(J, K, M, T)$.

The syndrome formers in the ensemble $\mathcal{C}(J, K, M, T)$ have memory $m_{\mathrm{s}}=a-1$, independent of $M$, while $b$ and $c$ depend on $M$. This ensemble of codes is different from the LDPCCCs considered in [3], [12], and [13], where the codes have varying syndrome former memories $m_{\mathrm{s}}$, while the rate parameters $b$ and $c$ are fixed. For the ensemble $\mathcal{C}(J, K, M, T)$, as $M$ increases, i.e., as $b$ and $c$ increase, the syndrome formers become increasingly sparse.

During the encoding process, the information sequences are divided into blocks of $b=$ $\left(K^{\prime}-J^{\prime}\right) M$ symbols, which are input to an LDPC convolutional encoder at each time instant 


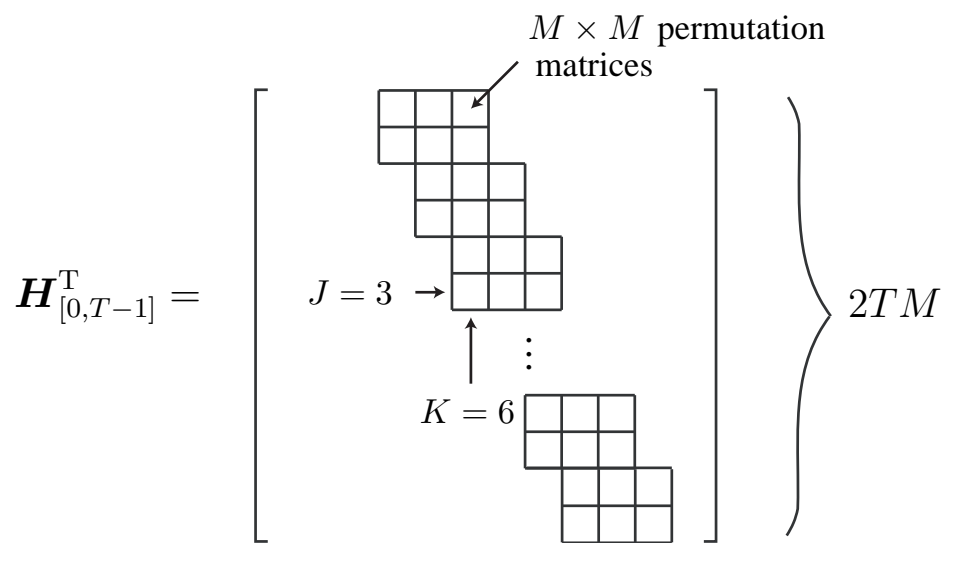

Fig. 1. One period of a syndrome former for a code in the ensemble $\mathcal{C}(3,6, M, T)$.

$t$, and a block of $c=K^{\prime} M$ encoded symbols is generated at the output. For any code in $\mathcal{C}(J, K, M, T)$, an equivalent systematic LDPC convolutional encoder can be constructed such that the computational complexity per encoded parity-check symbol depends only on $K$ and is independent of the permutation matrix size $M$ (see [3]).

Since there are at least $J^{\prime}$ linearly dependent columns in $\boldsymbol{H}_{0}^{\mathrm{T}}(t)$ for any code in $\mathcal{C}(J, K, M, T)$, $\boldsymbol{H}_{[0, T-1]}^{\mathrm{T}}$ defines a rate $R \geq 1-\frac{J^{\prime} M-\left(J^{\prime}-1\right)}{K^{\prime} M}$ code. The constraint length of codes from $\mathcal{C}(J, K, M, T)$ is defined ${ }^{3}$ as $\nu=\left(m_{\mathrm{s}}+1\right) \cdot c=a \cdot K^{\prime} M=K M$. For example, the codes in the ensemble $\mathcal{C}(3,6, M, T)$ have constraint length $\nu=6 M$.

For $T \geq J$, a syndrome former $\tilde{\boldsymbol{H}}_{[0, T-1]}^{\mathrm{T}}$ for a $(J, K)$ regular $T B$ - LDPCCC can be constructed from one period of a syndrome former $\boldsymbol{H}_{[0, T-1]}^{\mathrm{T}}$ for a $(J, K)$ regular $T$-periodic LDPCCC. This can be done by wrapping back the last $J-1$ blocks of columns of $\boldsymbol{H}_{[0, T-1]}^{\mathrm{T}}$. For the $(3,6)$ case, $\tilde{\boldsymbol{H}}_{[0, T-1]}^{\mathrm{T}}$ can be constructed from $\boldsymbol{H}_{[0, T-1]}^{\mathrm{T}}$ (see Fig. 1) for any $T \geq 3$, as illustrated in Fig. 2. TB-LDPCCC's created in this way form an ensemble which we denote by $\tilde{\mathcal{C}}(J, K, M, T)$. The block length of these codes is $2 T M$. In the following section we will use this ensemble to derive a lower bound on the minimum distance of TB-LDPCCCs.

\footnotetext{
${ }^{3} \mathrm{~A}$ discussion of the definition of constraint length for LDPCCCs is given in [1].
} 


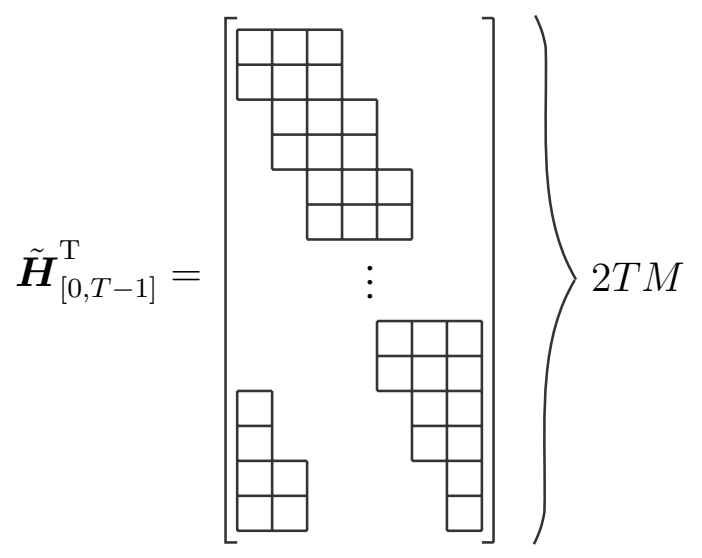

Fig. 2. Syndrome former of a TB-LDPCCC in the ensemble $\tilde{\mathcal{C}}(J, K, M, T)$

\section{A Lower Bound on the Minimum Distance of TB-LDPCCCS}

To avoid cumbersome notation, we focus on the $(3,6)$ regular TB-LDPCCC case, i.e., we consider the ensemble $\tilde{\mathcal{C}}(3,6, M, T)$, although the same technique can also be applied more generally.

Consider a length $2 T M$ vector $\tilde{\boldsymbol{v}}_{[0, T-1]}=\left(\tilde{\boldsymbol{v}}_{0}^{(0)}, \tilde{\boldsymbol{v}}_{0}^{(1)}, \ldots, \tilde{\boldsymbol{v}}_{T-1}^{(0)}, \tilde{\boldsymbol{v}}_{T-1}^{(1)}\right), \boldsymbol{v}_{t}^{(h)} \in \mathbb{F}_{2}^{M}$, where $\tilde{\boldsymbol{v}}_{t}^{(h)}=\left(\tilde{v}_{t 1}^{(h)}, \tilde{v}_{t 2}^{(h)}, \ldots, \tilde{v}_{t M}^{(h)}\right), t=0,1, \ldots, T-1, h=0,1$. A vector $\tilde{\boldsymbol{v}}_{[0, T-1]}$ is a codeword in a $(3,6)$ regular TB-LDPCCC iff it satisfies the $T M$ equations (constraints) defined by the syndrome former $\tilde{\boldsymbol{H}}_{[0, T-1]}^{\mathrm{T}}$, i.e.,

$$
\tilde{\boldsymbol{v}}_{[0, T-1]} \tilde{\boldsymbol{H}}_{[0, T-1]}^{\mathrm{T}}=\mathbf{0} .
$$

For the ensemble $\tilde{\mathcal{C}}(3,6, M, T)$, these $T M$ parity-check equations can be divided into $T$ sets where the $t$ th set $\mathcal{S}^{(t)}, t=0,1, \ldots, T-1$, consists of the $M$ parity-check equations determined by the six permutation matrices located in the th block of columns of the syndrome former $\tilde{\boldsymbol{H}}_{[0, T-1]}^{\mathrm{T}}$.

Let $\tilde{d}_{t}^{(h)}$ be the Hamming weight of the vector $\tilde{\boldsymbol{v}}_{t}^{(h)}, h=0,1, t=0,1, \ldots, T-1$. We then say that $\tilde{\boldsymbol{v}}_{[0, T-1]}$ has weight composition $\tilde{\boldsymbol{d}}_{[0, T-1]}=\left(\tilde{d}_{0}^{(0)}, \tilde{d}_{0}^{(1)}, \ldots, \tilde{d}_{T-1}^{(0)}, \tilde{d}_{T-1}^{(1)}\right)$, and the Hamming weight of the vector $\tilde{\boldsymbol{v}}_{[0, T-1]}$ with weight composition $\tilde{\boldsymbol{d}}_{[0, T-1]}$ is $\tilde{d}_{[0, T-1]}=\tilde{d}_{0}^{(0)}+\tilde{d}_{0}^{(1)}+\cdots+$ $\tilde{d}_{T-1}^{(0)}+\tilde{d}_{T-1}^{(1)}$. Now note that there exists $\prod_{t=0}^{T-1}\left(\begin{array}{c}M \\ \tilde{d}_{t}^{(0)}\end{array}\right) \prod_{t=0}^{T-1}\left(\begin{array}{c}M \\ \tilde{d}_{t}^{(1)}\end{array}\right)$ vectors $\tilde{\boldsymbol{v}}_{[0, T-1]}$ with weight composition $\tilde{\boldsymbol{d}}_{[0, T-1]}$. Our goal is to calculate the average number of codewords $\tilde{\boldsymbol{v}}_{[0, T-1]}$ with weight composition $\tilde{\boldsymbol{d}}_{[0, T-1]}$ for a code in the ensemble $\tilde{\mathcal{C}}(3,6, M, T)$. Finally, in the asymptotic 
case, as $M \rightarrow \infty$, it is more convenient to operate with the normalized weight composition $\tilde{\boldsymbol{\rho}}_{[0, T-1]}=\left(\tilde{\rho}_{0}^{(0)}, \tilde{\rho}_{0}^{(1)}, \ldots, \tilde{\rho}_{T-1}^{(0)}, \tilde{\rho}_{T-1}^{(1)}\right)$, where $\tilde{\rho}_{t}^{(h)}=\tilde{d}_{t}^{(h)} / M$.

Letting $\tilde{\rho}_{-2}^{(h)}=\tilde{\rho}_{T-2}^{(h)}$ and $\tilde{\rho}_{-1}^{(h)}=\tilde{\rho}_{T-1}^{(h)}$ for $h=0,1$, the probability that a vector $\tilde{\boldsymbol{v}}_{[0, T-1]}$ satisfies the $t$ th set of constraints, $\mathcal{S}^{(t)}, t=0,1, \ldots, T-1$, in the ensemble $\tilde{\mathcal{C}}(3,6, M, T)$ can be upper bounded (see [1], Appendix I) as

$$
P\left(\tilde{\boldsymbol{\rho}}_{[0, T-1]}, \mathcal{S}^{(t)}\right) \leq \frac{\exp \left[M \tilde{F}\left(\lambda_{1}^{(t)}, \lambda_{2}^{(t)}, \ldots, \lambda_{6}^{(t)}, \tilde{\rho}_{t-2}^{(0)}, \tilde{\rho}_{t-2}^{(1)}, \tilde{\rho}_{t-1}^{(0)}, \tilde{\rho}_{t-1}^{(1)}, \tilde{\rho}_{t}^{(0)}, \tilde{\rho}_{t}^{(1)}\right)\right]}{\prod_{i=t-2}^{t} \prod_{h=0}^{1}\left(\tilde{\rho}_{i}^{(h)} M\right)}
$$

where $\lambda_{1}^{(t)}, \lambda_{2}^{(t)}, \ldots, \lambda_{6}^{(t)}$ are arbitrary constants,

$$
\tilde{F}\left(\lambda_{1}, \lambda_{2}, \ldots, \lambda_{6}, \rho_{1}, \rho_{2}, \ldots, \rho_{6}\right) \stackrel{\text { def }}{=} g\left(\lambda_{1}, \lambda_{2}, \ldots, \lambda_{6}\right)-\sum_{k=1}^{6} \lambda_{k} \rho_{k}
$$

and

$$
g\left(\lambda_{1}, \lambda_{2}, \ldots, \lambda_{6}\right) \stackrel{\text { def }}{=} \ln \frac{\prod_{k=1}^{6}\left(1+e^{\lambda_{k}}\right)+\prod_{k=1}^{6}\left(1-e^{\lambda_{k}}\right)}{2} .
$$

We notice that there are $T$ independent constraint sets $\mathcal{S}^{(t)}, t=0,1, \ldots, T-1$, and the number of vectors $\tilde{\boldsymbol{v}}_{[0, T-1]}$ having normalized weight composition $\tilde{\boldsymbol{\rho}}_{[0, T-1]}$ is

$$
N\left(\tilde{\boldsymbol{\rho}}_{[0, T-1]}\right) \stackrel{\text { def }}{=} \prod_{t=0}^{T-1}\left(\begin{array}{c}
M \\
\tilde{\rho}_{t}^{(0)} M
\end{array}\right) \prod_{t=0}^{T-1}\left(\begin{array}{c}
M \\
\tilde{\rho}_{t}^{(1)} M
\end{array}\right) .
$$

Thus the expected number of vectors with normalized weight composition $\tilde{\boldsymbol{\rho}}_{[0, T-1]}$ satisfying all $T$ sets of constraints, i.e., vectors that are codewords in the TB-LDPCCCC code defined by $\tilde{\boldsymbol{H}}_{[0, T-1]}^{\mathrm{T}}$, is given by

$$
E\left(\tilde{\boldsymbol{\rho}}_{[0, T-1]}\right) \stackrel{\text { def }}{=} N\left(\tilde{\boldsymbol{\rho}}_{[0, T-1]}\right) \prod_{t=0}^{T-1} P\left(\tilde{\boldsymbol{\rho}}_{[0, T-1]}, \mathcal{S}^{(t)}\right) .
$$

Substituting (6) into (10), we obtain the upper bound

$$
E\left(\tilde{\boldsymbol{\rho}}_{[0, T-1]}\right) \leq \frac{\exp \left[M \sum_{t=0}^{T-1} \tilde{F}\left(\lambda_{1}^{(t)}, \lambda_{2}^{(t)}, \ldots, \lambda_{6}^{(t)}, \tilde{\rho}_{t-2}^{(0)}, \tilde{\rho}_{t-2}^{(1)}, \ldots, \tilde{\rho}_{t}^{(1)}\right)\right]}{\left(\prod_{t=0}^{T-1}\left(\begin{array}{c}
M \\
\tilde{\rho}_{t}^{(0)} M
\end{array}\right) \prod_{t=0}^{T-1}\left(\tilde{\rho}_{t}^{(1)} M\right)\right)^{2}}
$$


where we recall that $\tilde{\rho}_{-2}^{(h)}=\tilde{\rho}_{T-2}^{(h)}$ and $\tilde{\rho}_{-1}^{(h)}=\tilde{\rho}_{T-1}^{(h)}, h=0,1$. Now using Lemma 1 and Appendix II from [1], we can further upper bound $E\left(\tilde{\boldsymbol{\rho}}_{[0, T-1]}\right)$ as

$$
\begin{aligned}
E\left(\tilde{\boldsymbol{\rho}}_{[0, T-1]}\right) & \leq\left[\prod_{t=0}^{T-1} \sigma\left(\tilde{\rho}_{t}^{(0)}, M\right) \prod_{t=0}^{T-1} \sigma\left(\tilde{\rho}_{t}^{(1)}, M\right)\right]^{2} \\
& \cdot \exp \left[M \sum_{t=0}^{T-1} F\left(\lambda_{1}^{(t)}, \lambda_{2}^{(t)}, \ldots, \lambda_{6}^{(t)}, \tilde{\rho}_{t-2}^{(0)}, \tilde{\rho}_{t-2}^{(1)}, \ldots, \tilde{\rho}_{t}^{(1)}\right)\right],
\end{aligned}
$$

where

$$
\begin{gathered}
F\left(\lambda_{1}, \lambda_{2}, \ldots, \lambda_{6}, \rho_{1}, \rho_{2}, \ldots, \rho_{6}\right) \stackrel{\text { def }}{=} \tilde{F}\left(\lambda_{1}, \lambda_{2}, \ldots, \lambda_{6}, \rho_{1}, \rho_{2}, \ldots, \rho_{6}\right)-\frac{2}{3} \sum_{k=1}^{6} H\left(\rho_{k}\right), \\
\sigma(\rho, M) \stackrel{\text { def }}{=}\left\{\begin{array}{cc}
1, & \text { if } \rho=0, \\
\sqrt{12 M \rho(1-\rho)}, & \text { otherwise, }
\end{array}\right.
\end{gathered}
$$

and

$$
H(\rho) \stackrel{\text { def }}{=}-\rho \ln \rho-(1-\rho) \ln (1-\rho)
$$

Now suppose that for a particular normalized weight composition $\tilde{\boldsymbol{\rho}}_{[0, T-1]}$, there exists a set $\left\{\lambda_{1}^{(0)}, \lambda_{2}^{(0)}, \ldots, \lambda_{6}^{(0)}, \ldots, \lambda_{1}^{(T-1)}, \lambda_{2}^{(T-1)}, \ldots, \lambda_{6}^{(T-1)}\right\}$ of $6 T$ coefficients such that the sum of the functions $\sum_{t=0}^{T-1} F\left(\lambda_{1}^{(t)}, \lambda_{2}^{(t)}, \ldots, \lambda_{6}^{(t)}, \tilde{\rho}_{t-2}^{(0)}, \tilde{\rho}_{t-2}^{(1)}, \ldots, \tilde{\rho}_{t}^{(1)}\right)$ is negative. Then the average number of codewords having normalized weight composition $\tilde{\boldsymbol{\rho}}_{[0, T-1]}$ goes to zero exponentially as $M$ tends to infinity. The tightest bound on $E\left(\tilde{\boldsymbol{\rho}}_{[0, T-1]}\right)$ can be obtained by minimizing each function $F\left(\lambda_{1}^{(t)}, \lambda_{2}^{(t)}, \ldots, \lambda_{6}^{(t)}, \tilde{\rho}_{t-2}^{(0)}, \tilde{\rho}_{t-2}^{(1)}, \ldots, \tilde{\rho}_{t}^{(1)}\right), t=0,1, \ldots, T-1$, with respect to its parameters $\lambda_{1}^{(t)}, \lambda_{2}^{(t)}, \ldots, \lambda_{6}^{(t)}$.

We begin by defining

$$
G\left(\rho_{1}, \rho_{2}, \ldots, \rho_{6}\right) \stackrel{\text { def }}{=} \min _{\lambda_{1}, \lambda_{2}, \ldots, \lambda_{6}} F\left(\lambda_{1}, \lambda_{2}, \ldots, \lambda_{6}, \rho_{1}, \rho_{2}, \ldots, \rho_{6}\right) .
$$

Then from (12) we obtain

$$
E\left(\tilde{\boldsymbol{\rho}}_{[0, T-1]}\right) \leq\left[\prod_{t=0}^{T-1} \sigma\left(\tilde{\rho}_{t}^{(0)}, M\right) \prod_{t=0}^{T-1} \sigma\left(\tilde{\rho}_{t}^{(1)}, M\right)\right]^{2} \exp \left[M \sum_{t=0}^{T-1} G\left(\tilde{\rho}_{t-2}^{(0)}, \tilde{\rho}_{t-2}^{(1)}, \ldots, \tilde{\rho}_{t}^{(1)}\right)\right]
$$

or alternatively

$$
E\left(\tilde{\boldsymbol{\rho}}_{[0, T-1]}\right) \leq\left[\prod_{t=0}^{T-1} \sigma\left(\rho_{t}^{(0)}, M\right) \prod_{t=0}^{T-1} \sigma\left(\rho_{t}^{(1)}, M\right)\right]^{2} \exp \left[M G\left(\tilde{\boldsymbol{\rho}}_{[0, T-1]}\right)\right]
$$


where

$$
G\left(\tilde{\boldsymbol{\rho}}_{[0, T-1]}\right) \stackrel{\text { def }}{=} \sum_{t=0}^{T-1} G\left(\tilde{\rho}_{t-2}^{(0)}, \tilde{\rho}_{t-2}^{(1)}, \ldots, \tilde{\rho}_{t}^{(1)}\right)
$$

is a function of the $2 T$-dimensional normalized weight vector $\tilde{\boldsymbol{\rho}}_{[0, T-1]}=\left(\tilde{\rho}_{0}^{(0)}, \tilde{\rho}_{0}^{(1)}, \ldots, \tilde{\rho}_{T-1}^{(0)}, \tilde{\rho}_{T-1}^{(1)}\right)$.

We now let $\tilde{\rho}_{[0, T-1]}=\frac{1}{2 T}\left(\sum_{t=0}^{T-1} \tilde{\rho}_{t}^{(0)}+\sum_{t=0}^{T-1} \tilde{\rho}_{t}^{(1)}\right)$ be the normalized Hamming weight of the vector $\tilde{\boldsymbol{\rho}}_{[0, T-1]}$. If the function $G\left(\tilde{\boldsymbol{\rho}}_{[0, T-1]}\right)$ is negative for all $\tilde{\boldsymbol{\rho}}_{[0, T-1]}$ with normalized weight $\tilde{\rho}_{[0, T-1]}=\rho_{0}$, then the corresponding mathematical expectation $E\left(\tilde{\boldsymbol{\rho}}_{[0, T-1]}\right)$ goes to zero exponentially with $M$ as $M$ tends to infinity ${ }^{4}$.

The total number of weight compositions $\tilde{\boldsymbol{d}}_{[0, T-1]}=M \tilde{\boldsymbol{\rho}}_{[0, T-1]}$ is upper bounded by $(M+1)^{2 T}$. Hence, if $G\left(\tilde{\boldsymbol{\rho}}_{[0, T-1]}\right)<0$ for a $\tilde{\boldsymbol{\rho}}_{[0, T-1]}$ having normalized weight $\tilde{\rho}_{[0, T-1]} \leq \rho^{*}$, and $T$ is finite, the average number of nonzero codewords having weight $\tilde{d}_{[0, T-1]} \leq 2 \rho^{*} M T$ tends to zero as $M$ tends to infinity ${ }^{4}$.

Note that any code in $\tilde{\mathcal{C}}(3,6, M, T)$ always has codewords $\tilde{\boldsymbol{v}}_{[0, T-1]}=\left(\tilde{\boldsymbol{v}}_{0}^{(0)}, \tilde{\boldsymbol{v}}_{0}^{(1)}, \ldots, \tilde{\boldsymbol{v}}_{T-1}^{(0)}, \tilde{\boldsymbol{v}}_{T-1}^{(1)}\right)$ with $\tilde{\boldsymbol{v}}_{t}^{(0)}=\tilde{\boldsymbol{v}}_{t}^{(1)}=\mathbf{1}$ for some $t$, where $\mathbf{1}$ is the $M$-dimensional all-ones vector, and the remaining $2 T-2$ components of $\tilde{\boldsymbol{v}}_{[0, T-1]}$ equal to the $M$-dimensional all-zero vector. Such a codeword $\tilde{\boldsymbol{v}}_{[0, T-1]}$ has weight $2 M$, and this is an upper bound on the minimum distance of codes in $\tilde{\mathcal{C}}(3,6, M, T)$. Hence, it is sufficient to look at weight compositions with Hamming weight $\tilde{d}_{[0, T-1]}<2 M$, i.e., $\rho_{[0, T-1]}<\frac{1}{T}$.

We now summarize the arguments above in the following theorem:

Theorem 1: Suppose that the function $G\left(\tilde{\boldsymbol{\rho}}_{[0, T-1]}\right)$ is negative for all $\tilde{\boldsymbol{\rho}}_{[0, T-1]}$ of normalized weight $\tilde{\rho}_{[0, T-1]} \leq \rho^{*}$. Then, in the ensemble $\tilde{\mathcal{C}}(J, K, M, T)$, there exists a TB-LDPCCC with minimum distance $\tilde{d}_{[0, T-1]}$ lower bounded by

$$
\tilde{d}_{[0, T-1]} \geq \min \left\{2 M T \rho^{*}, 2 M\right\} .
$$

The parameter $\rho^{*}$ can be calculated numerically. The results of this calculation are presented in Section V. There we will see that, analogous to conventional tail-biting convolutional codes, for relatively small $T$ the inter minimum distance $d_{\text {inter }}$ determines the minimum distance of the TB-LDPCCC, while for larger $T$ the intra minimum distance $d_{\text {intra }}$, i.e., the free distance of the $T$-periodic LDPCCC, determines the minimum distance of the TB-LDPCCC.

${ }^{4}$ See [1] for a mathematically precise formulation of this result. 
In the following section, we show how this existence bound on the minimum distance of TB-LDPCCCs leads to an existence bound on the free distance of the T-periodic LDPCCCs.

\section{A LOWER Bound On THE FreE DistanCE OF T-PERIODIC LDPCCCS}

We begin by considering TB-LDPCCCs of length $2 \kappa T M$, where $\kappa$ is an integer satisfying $\kappa \geq 1$. Any codeword $\tilde{\boldsymbol{v}}_{[0, \kappa T-1]}$ in this code satisfies

$$
\tilde{\boldsymbol{v}}_{[0, \kappa T-1]} \tilde{\boldsymbol{H}}_{[0, \kappa T-1]}^{\mathrm{T}}=\mathbf{0},
$$

where the transposed parity-check matrix $\tilde{\boldsymbol{H}}_{[0, \kappa T-1]}^{\mathrm{T}}$ of the TB-LDPCCC is constructed from a syndrome former $\boldsymbol{H}_{[0, \kappa T-1]}^{\mathrm{T}}$ of a $T$-periodic LDPCCC by wrapping back the last two blocks of columns (see Section II). The product of a codeword $\tilde{\boldsymbol{v}}_{[0, \kappa T-1]}$ in the length $2 \kappa T M$ TBLDPCCC and the syndrome former $\boldsymbol{H}_{[0, \kappa T-1]}^{\mathrm{T}}$ of the $T$-periodic LDPCCC defines an $M(\kappa T+2)$ dimensional syndrome vector

$$
\boldsymbol{s}_{[0, \kappa T+1]}=\tilde{\boldsymbol{v}}_{[0, \kappa T-1]} \boldsymbol{H}_{[0, \kappa T-1]}^{\mathrm{T}},
$$

where the syndrome vector

$$
\boldsymbol{s}_{[0, \kappa T+1]}=\left(\boldsymbol{s}_{0}, \boldsymbol{s}_{1}, \ldots, \boldsymbol{s}_{\kappa T+1}\right)
$$

is a concatenation of $M$-dimensional subvectors $\boldsymbol{s}_{t}=\left(s_{t 1}, s_{t 2}, \ldots, s_{t M}\right), t=0,1, \ldots, \kappa T+1$. Since $\tilde{\boldsymbol{v}}_{[0, \kappa T-1]}$ satisfies (20) and $\tilde{\boldsymbol{H}}_{[0, \kappa T-1]}^{\mathrm{T}}$ is constructed from $\boldsymbol{H}_{[0, \kappa T-1]}^{\mathrm{T}}$ using the wrapping back procedure of Fig. 2, the subvectors $s_{t}$ satisfy the conditions

$$
\begin{aligned}
& \boldsymbol{s}_{0}=\boldsymbol{s}_{\kappa T}, \\
& \boldsymbol{s}_{1}=\boldsymbol{s}_{\kappa T+1},
\end{aligned}
$$

and

$$
\boldsymbol{s}_{t}=\mathbf{0}, \quad t=2,3, \ldots, \kappa T-1
$$

Therefore (20), which defines the codewords of the TB-LDPCCC, is equivalent to (21), where the syndrome vector $\boldsymbol{s}_{[0, \kappa T+1]}$ satisfies conditions (23)-(25).

Lemma 1: Let $\tilde{d}_{[0, \kappa T-1]}$, for any integer $\kappa \geq 1$, be the minimum distance of the TB-LDPCCC defined by (20). Then there exists $\kappa_{0}>0$ such that for any $\kappa \geq \kappa_{0}$ the free distance $d_{\text {free }}$ of the $T$-periodic LDPCCC with syndrome former $\boldsymbol{H}_{[0, \kappa T-1]}^{\mathrm{T}}$ is lower bounded by

$$
d_{\text {free }} \geq \tilde{d}_{[0, \kappa T-1]}
$$


Proof: The proof follows from the definitions of free distance and row distance. The $L$ th order row distance $d_{L}^{r}$ of a periodically time-varying convolutional code is defined [11] as the minimum weight of all code sequences having a nonzero segment of length at most $L+m+1$ (in this case, the code sequences are composed of blocks of length $2 M$ ), where $m$ is the encoder memory. In turn, the free distance $d_{\text {free }}$ is defined as

$$
d_{\text {free }}=\min _{L} d_{L}^{r}
$$

where $d_{L}^{r}$ is monotonically decreasing with $L$ and there exists an integer $L_{0}$ such that

$$
d_{L}^{r}=d_{L_{0}}^{r}=d_{\text {free }}
$$

for any $L>L_{0}$.

Thus, we can find a sufficiently large $\kappa_{0}$ such that, for any $\kappa \geq \kappa_{0}$, the code sequences of the length $2 \kappa T M$ tail-biting code include all possible nonzero segments of length $L_{0}+m+1$ blocks of the $T$-periodic convolutional code. This implies that

$$
d_{L_{0}}^{r} \geq \tilde{d}_{[0, \kappa T-1]}
$$

which, along with (28), leads to (26).

Now let $\tilde{\boldsymbol{v}}_{[0, \kappa T-1]}=\left(\tilde{\boldsymbol{v}}_{0}^{(0)}, \tilde{\boldsymbol{v}}_{0}^{(1)}, \ldots, \tilde{\boldsymbol{v}}_{\kappa T-1}^{(0)}, \tilde{\boldsymbol{v}}_{\kappa T-1}^{(1)}\right)$ be a codeword in the length $2 \kappa T M$ TBLDPCCC, i.e., it satisfies (20). Note that this codeword can be represented as

$$
\tilde{\boldsymbol{v}}_{[0, \kappa T-1]}=\left(\tilde{\boldsymbol{v}}_{[0, T-1]}, \tilde{\boldsymbol{v}}_{[T, 2 T-1]}, \ldots, \tilde{\boldsymbol{v}}_{[(\kappa-1) T, \kappa T-1]}\right),
$$

where

$$
\tilde{\boldsymbol{v}}_{[(i-1) T, i T-1]}=\left(\tilde{\boldsymbol{v}}_{(i-1) T}^{(0)}, \tilde{\boldsymbol{v}}_{(i-1) T}^{(1)}, \ldots, \tilde{\boldsymbol{v}}_{i T-1}^{(0)}, \tilde{\boldsymbol{v}}_{i T-1}^{(1)}\right), \quad i=1, \ldots, \kappa
$$

Then consider the sequence

$$
\overline{\boldsymbol{v}}_{[0, T-1]} \stackrel{\text { def }}{=} \tilde{\boldsymbol{v}}_{[0, T-1]}+\tilde{\boldsymbol{v}}_{[T, 2 T-1]}+\ldots+\tilde{\boldsymbol{v}}_{[(\kappa-1) T, \kappa T-1]}
$$

i.e., the modulo- 2 sum of the components of the codeword $\tilde{\boldsymbol{v}}_{[0, \kappa T-1]}$ given in (30). The following lemma proves that $\overline{\boldsymbol{v}}_{[0, T-1]}$ is a codeword in the TB-LDPCCC consisting of only one period of the $T$-periodic LDPCCC.

Lemma 2: The sequence $\overline{\boldsymbol{v}}_{[0, T-1]}$ satisfies

$$
\overline{\boldsymbol{v}}_{[0, T-1]} \tilde{\boldsymbol{H}}_{[0, T-1]}^{\mathrm{T}}=\mathbf{0},
$$


i.e., it is a code sequence of the TB-LDPCCC with length equal to one period of the $T$-periodic LDPCCC.

Proof: Equality (33) is equivalent (see (21)) to the equality

$$
\overline{\boldsymbol{v}}_{[0, T-1]} \boldsymbol{H}_{[0, T-1]}^{\mathrm{T}}=\boldsymbol{s}_{[0, T+1]}=\left(\boldsymbol{s}_{0}, \boldsymbol{s}_{1}, \ldots, \boldsymbol{s}_{T+1}\right),
$$

where

$$
\begin{aligned}
& \boldsymbol{s}_{0}=\boldsymbol{s}_{T}, \\
& \boldsymbol{s}_{1}=\boldsymbol{s}_{T+1},
\end{aligned}
$$

and

$$
\boldsymbol{s}_{t}=\mathbf{0}, \quad t=2,3, \ldots, T-1 .
$$

Therefore, instead of proving (33), we can alternatively prove that the syndrome vectors in (34) satisfy conditions (35)-(37). We begin by defining

$$
\tilde{\boldsymbol{v}}_{[(i-1) T, i T-1]} \boldsymbol{H}_{[0, T-1]}^{\mathrm{T}} \stackrel{\text { def }}{=} \boldsymbol{s}_{[0, T+1]}^{(i)}=\left(\boldsymbol{s}_{0}^{(i)}, \boldsymbol{s}_{1}^{(i)}, \ldots, \boldsymbol{s}_{T+1}^{(i)}\right), \quad i=1,2, \ldots, \kappa .
$$

Then it follows from (21) and (38) that the syndrome vector $\boldsymbol{s}_{[0, \kappa T+1]}$ of the length $2 \kappa T M$ TB-LDPCCC satisfies

$$
\begin{aligned}
& \left(\boldsymbol{s}_{0}, \boldsymbol{s}_{1}, \ldots, \boldsymbol{s}_{\kappa T+1}\right)=\left(\boldsymbol{s}_{0}^{(1)}, \boldsymbol{s}_{1}^{(1)}, \ldots, \boldsymbol{s}_{T-1}^{(1)}, \boldsymbol{s}_{T}^{(1)}, \boldsymbol{s}_{T+1}^{(1)}, \mathbf{0}, \mathbf{0}, \ldots, \quad \mathbf{0}, \quad \mathbf{0}\right. \\
& +\left(\mathbf{0}, \mathbf{0}, \ldots, \mathbf{0}, \boldsymbol{s}_{0}^{(2)}, \boldsymbol{s}_{1}^{(2)}, \boldsymbol{s}_{2}^{(2)}, \ldots, \boldsymbol{s}_{T-1}^{(2)}, \boldsymbol{s}_{T}^{(2)}, \boldsymbol{s}_{T+1}^{(2)}, \mathbf{0}, \ldots,\right.
\end{aligned}
$$

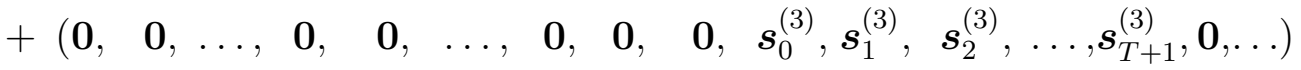

$$
\begin{aligned}
& +\ldots \text {, }
\end{aligned}
$$

where the addition is modulo 2 .

Since $\tilde{\boldsymbol{v}}_{[0, \kappa T-1]}$ ia a codeword in the length $2 \kappa T M$ TB-LDPCCC, conditions (23)-(25) are satisfied for the syndrome vector $\boldsymbol{s}_{[0, \kappa T+1]}$. This, together with (39), implies that the vectors $\boldsymbol{s}_{t}^{(i)}$ should satisfy

$$
\begin{aligned}
\boldsymbol{s}_{0}^{(1)} & =\boldsymbol{s}_{T}^{(\kappa)}, \\
\boldsymbol{s}_{1}^{(1)}=\boldsymbol{s}_{T+1}^{(\kappa)}, & \\
\boldsymbol{s}_{0}^{(i)}=\boldsymbol{s}_{T}^{(i-1)}, & i=2,3, \ldots, \kappa, \\
\boldsymbol{s}_{1}^{(i)}=\boldsymbol{s}_{T+1}^{(i-1)}, & i=2,3, \ldots, \kappa,
\end{aligned}
$$


and

$$
\boldsymbol{s}_{t}^{(i)}=\mathbf{0}, \quad t=2,3, \ldots, T-1, i=1,2, \ldots, \kappa
$$

From the definition of $\overline{\boldsymbol{v}}_{[0, T-1]}$ in (32), the definition of its syndrome vector $\boldsymbol{s}_{[0, T+1]}$ in (34), and the definition of $\boldsymbol{s}_{[0, T+1]}^{(i)}$ in (38), it follows that

$$
\boldsymbol{s}_{[0, T+1]}=\boldsymbol{s}_{[0, T+1]}^{(1)}+\boldsymbol{s}_{[0, T+1]}^{(2)}+\ldots+\boldsymbol{s}_{[0, T+1]}^{(\kappa)},
$$

where the addition is modulo 2. It can be checked that (40)-(45) imply (35)-(37), and hence the lemma is proved.

The next lemma relates the minimum distances of the length $2 \kappa T M$ TB-LDPCCC and the length $2 T M$ TB-LDPCCC constructed from the same $T$-periodic LDPCCC.

Lemma 3: For any positive integer $\kappa$, the minimum distance $\tilde{d}_{[0, \kappa T-1]}$ of the length $2 \kappa T M$ TB-LDPCCC defined by (20) is lower bounded by the minimum distance $\tilde{d}_{[0, T-1]}$ of the length $2 T M$ TB-LDPCCC, i.e., $\tilde{d}_{[0, \kappa T-1]} \geq \tilde{d}_{[0, T-1]}$.

Proof: For any codeword $\tilde{\boldsymbol{v}}_{[0, \kappa T-1]}$ in the length $2 \kappa T M$ TB-LDPCCC represented by (30),

$$
w_{\mathrm{H}}\left(\tilde{\boldsymbol{v}}_{[0, \kappa T-1]}\right)=w_{\mathrm{H}}\left(\tilde{\boldsymbol{v}}_{[0, T-1]}\right)+w_{\mathrm{H}}\left(\tilde{\boldsymbol{v}}_{[T, 2 T-1]}\right)+\cdots+w_{\mathrm{H}}\left(\tilde{\boldsymbol{v}}_{[(\kappa-1) T, \kappa T-1]}\right),
$$

where $w_{\mathrm{H}}(\cdot)$ is the Hamming weight operator. From (32) and the triangle inequality it then follows that

$$
w_{\mathrm{H}}\left(\overline{\boldsymbol{v}}_{[0, T-1]}\right) \leq w_{\mathrm{H}}\left(\tilde{\boldsymbol{v}}_{[0, T-1]}\right)+\ldots+w_{\mathrm{H}}\left(\tilde{\boldsymbol{v}}_{[(\kappa-1) T, \kappa T-1]}\right)
$$

Since from Lemma 2 we know that $\overline{\boldsymbol{v}}_{[0, T-1]}$ is a codeword in the length $2 T M$ TB-LDPCCC, it follows that

$$
\tilde{d}_{[0, T-1]} \leq w_{\mathrm{H}}\left(\overline{\boldsymbol{v}}_{[0, T-1]}\right)
$$

Combining (46)-(48) we obtain for the codeword $\tilde{\boldsymbol{v}}_{[0, \kappa T-1]}$ of the length $2 \kappa T M$ TB-LDPCCC that

$$
\tilde{d}_{[0, T-1]} \leq w_{\mathrm{H}}\left(\tilde{\boldsymbol{v}}_{[0, \kappa T-1]}\right)
$$

which directly implies

$$
\tilde{d}_{[0, T-1]} \leq \tilde{d}_{[0, \kappa T-1]}
$$

and the lemma is proved.

Theorem 2 now follows directly from Lemmas 1 and 3. 
Theorem 2: The free distance $d_{\text {free }}$ of any $T$-periodic LDPCCC from the ensemble $\mathcal{C}(3,6, M, T)$ is lower bounded by the minimum distance of the corresponding TB-LDPCCC of block length $2 T M$, i.e.,

$$
d_{\text {free }} \geq \tilde{d}_{[0, T-1]}
$$

The distance properties of convolutional codes are characterized by the ratio $d_{\text {free }} / \nu$. In Section $\mathrm{V}$, we use Theorems 1 and 2 to numerically calculate the lower bound $\alpha_{\mathrm{LDPCCC}}=$ $\tilde{d}_{[0, T-1]} / \nu$ on $d_{\text {free }} / \nu$.

\section{Numerical Results}

Consider the function $G\left(\tilde{\boldsymbol{\rho}}_{[0, T-1]}\right)$ defined in Section III. According to the condition of Theorem 1, we must find the maximum $\rho_{[0, T-1]}^{*}$ such that $G\left(\tilde{\boldsymbol{\rho}}_{[0, T-1]}\right)<0$ for all $\tilde{\boldsymbol{\rho}}_{[0, T-1]}$ with normalized weight $\tilde{\rho}_{[0, T-1]} \leq \rho_{[0, T-1]}^{*}$.

The numerical procedure used to find $\rho_{[0, T-1]}^{*}$ can be outlined as follows. For a given $\tilde{\rho}_{[0, T-1]}$, we find the vector $\tilde{\boldsymbol{\rho}}_{[0, T-1]}$ with normalized weight $\tilde{\rho}_{[0, T-1]}$ that maximizes the function $G\left(\tilde{\boldsymbol{\rho}}_{[0, T-1]}\right)$ using numerical optimization. If $G\left(\tilde{\boldsymbol{\rho}}_{[0, T-1]}\right)>0$, we decrease $\tilde{\rho}_{[0, T-1]}$; otherwise (i.e., if $\left.G\left(\tilde{\boldsymbol{\rho}}_{[0, T-1]}\right)<0\right)$ we increase $\tilde{\rho}_{[0, T-1]}$ and repeat the procedure until the function $G\left(\tilde{\boldsymbol{\rho}}_{[0, T-1]}\right)$ becomes less than, for example, $-10^{-5}$. The end result of the procedure is a vector $\tilde{\boldsymbol{\rho}}_{[0, T-1]}^{*}=$ $\left(\tilde{\rho}_{0}^{(0)}, \tilde{\rho}_{0}^{(1)}, \ldots, \tilde{\rho}_{T-1}^{(0)}, \tilde{\rho}_{T-1}^{(1)}\right)$ and its normalized weight $\rho_{[0, T-1]}^{*}=\frac{1}{2 T}\left(\sum_{t=0}^{T-1} \tilde{\rho}_{t}^{(0)}+\sum_{t=0}^{T-1} \tilde{\rho}_{t}^{(1)}\right)$.

In Fig. 3, the resulting maximizing vectors $\tilde{\boldsymbol{\rho}}_{[0, T-1]}^{*}$ are shown for $T=5,12,13$. In particular, for $T=5$, we have

$$
\tilde{\boldsymbol{\rho}}_{[0, T-1]}^{*}=(0.023,0.023, \ldots, 0.023)
$$

The normalized weight $\tilde{\rho}_{[0, T-1]}=0.023$ of this vector is close to Gallager's lower bound on the minimum distance to block length ratio of LDPC block codes derived in [2]. This confirms the general rule that, for relatively small block lengths, the inter minimum distance $d_{\text {inter, }}$, which reflects the block code properties of tail-biting convolutional codes, determines their minimum distance. Indeed, the same tendency is observed for $T=3,4, \ldots, 11$, i.e., each component of the maximizing vector is approximately equal to 0.023 , resulting in a normalized weight of 0.023 .

For $T=11$, we see from (52) that the sum of the components of the maximizing vector $\tilde{\boldsymbol{\rho}}_{[0, T-1]}^{*}$ is approximately 0.5 . The same is also true for $T=12$ and $T=13$ (even though as shown in 


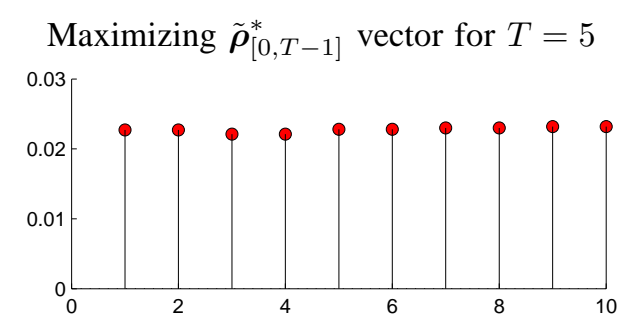

(a)

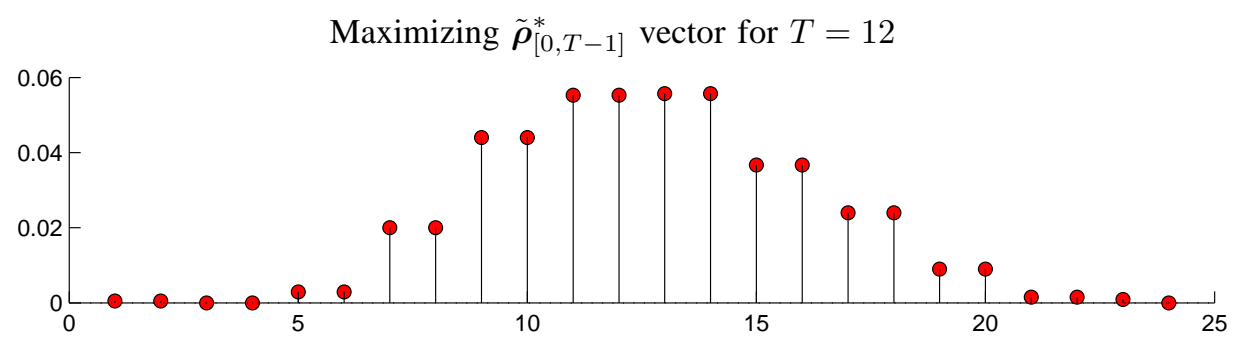

(b)

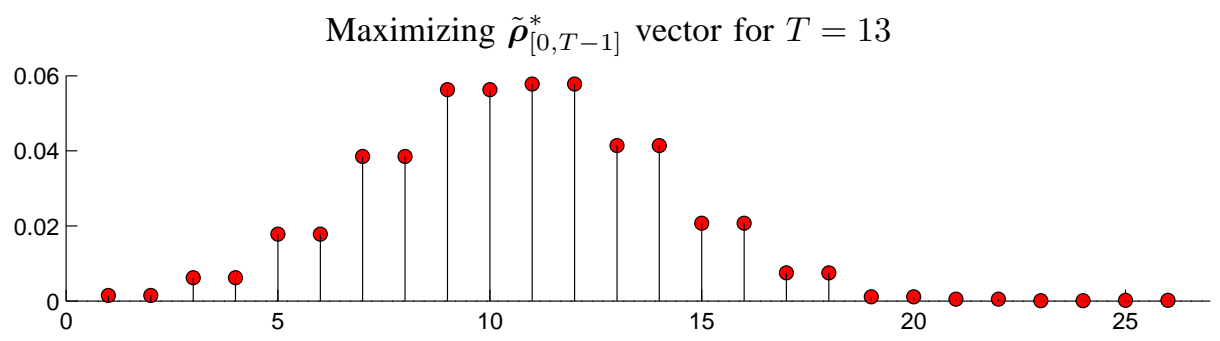

(c)

Fig. 3. Maximizing $\tilde{\boldsymbol{\rho}}_{[0, T-1]}^{*}$ vectors for $T=5,12,13$.

Figs. 3 (b) and 3 (c), the components of the maximizing vector are no longer approximately equal in these cases), and further increases in the period do not lead to higher values of this sum. It follows that the normalized weight drops as $T$ increases beyond 11 . This effect is observed due to the intra minimum distance $d_{\text {intra }}$, which is lower bounded by the bound on free distance of LDPCCCs derived in [1]. This bound scales as $\alpha_{\text {LDPCCC }}(3,6) \nu=6 \alpha_{\text {LDPCCC }}(3,6) M \approx 0.5 M$ and represents an upper bound on the minimum distance of TB-LDPCCCs. In other words, the minimum distance to block length ratio of TB-LDPCCCs decreases as $T$ increases beyond 11 , since the block length continues to increase while the minimum distance cannot grow beyond the constant $d_{\text {intra }}$. 
The distance ratios for the various LDPCCC ensembles are presented in Fig 4. We see that the minimum distance to block length ratio for TB-LDPCCCs is equal to Gallager's ratio for LDPC block codes when the period $T$ is small. For larger periods, however, the ratio drops and tends to zero, due to the effect of $d_{\text {intra }}$, as noted above. On the other hand, the free distance to constraint length ratio for $T$-periodic LDPCCCs grows with increasing $T$ and approaches the ratio derived in [1] for general (non-periodic) time-varying LDPCCCs as $T$ increases beyond 11.

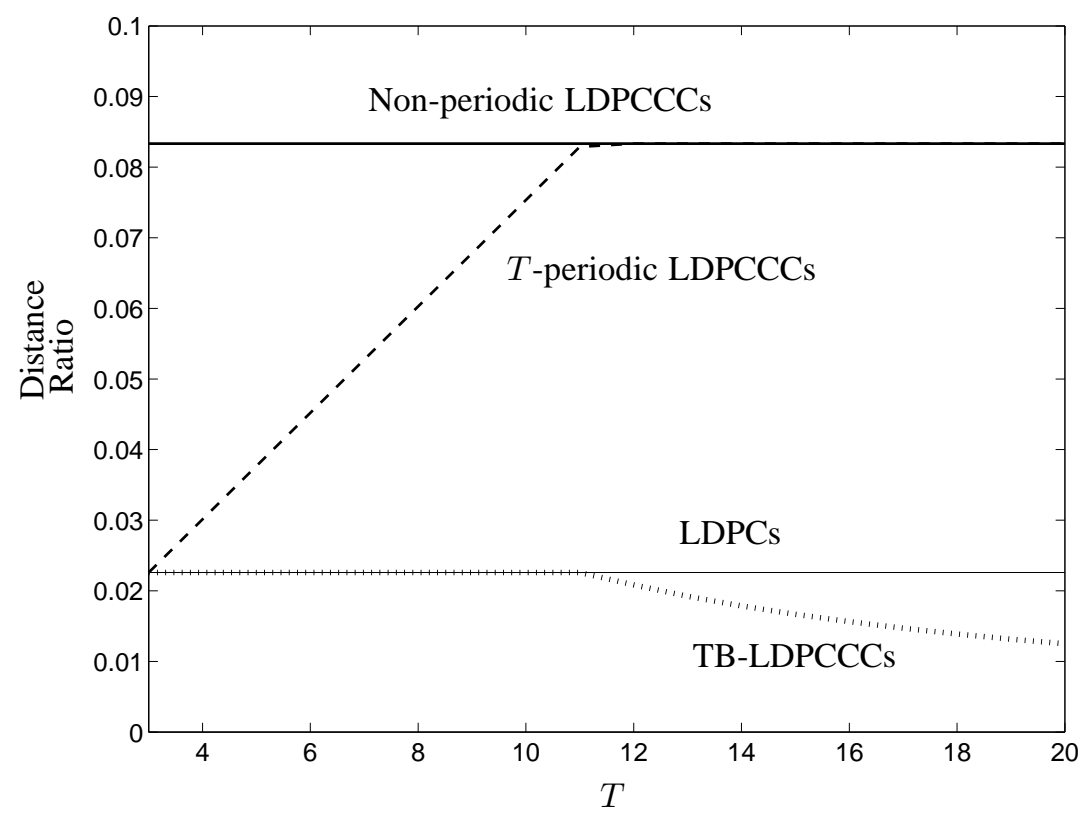

Fig. 4. Distance ratios for $T$-periodic LDPCCCs and TB-LDPCCCs as a function of the period $T$.

\section{CONCLUSIONS}

In this paper, we derived a lower bound on the free distance of periodically time-varying $(J, K)$ regular LDPCCCs and a lower bound on the minimum distance of the associated TBLDPCCCs. Theorems 1 and 2 give analytical expressions for these bounds in the general case. Using these expressions, we calculated numerically the bounds on free distance and minimum distance for the practically interesting $(3,6)$ regular LDPCCC case. In the limiting cases, for $T>11$ the free distance bound corresponds to the bound for general (non-periodic) time-varying 
LDPCCCs derived in [1], and for $T=3$ the minimum distance bound corresponds to Gallager's bound for LDPC block codes.

\section{REFERENCES}

[1] A. Sridharan, D. V. Truhachev, M. Lentmaier, D. J. Costello, Jr., and K. Sh. Zigangirov, "Distance bounds for an ensemble of LDPC convolutional codes", IEEE Trans. on Inform. Theory, IT-53(12):4537-4555, Dec. 2007.

[2] R. G. Gallager, Low-density parity-check codes. M.I.T. Press, Cambridge, Massachusetts, 1963.

[3] A. Jimenez-Felström and K. S. Zigangirov, "Time-varying convolutional codes with low-density parity-check matrix", IEEE Trans. on Inform. Theory, vol. IT-45, no. 5, pp. 2181-2190, Sept.1999.

[4] E. N. Gilbert, “A comparison of signalling alphabets,” Bell System Technical Journal, vol. 31, pp. 504-522, 1952.

[5] R. R. Varsharmov, "Estimates of the number of signals in error correcting codes," Doklady A.N.S.S.R, vol. 117, no. 5, pp. 739-741, 1957.

[6] D. J. Costello, Jr., "Free distance bounds for convolutional codes," IEEE Transactions on Information Theory, vol. IT-20, no. 3, pp. 356-365, May 1974.

[7] R. M. Tanner, "Error-correcting coding system”, U.S. Patent No. 4295218, Oct. 1981.

[8] M. B. S. Tavares, K. Sh. Zigangirov, and G. P. Fettweis, "Tail-biting LDPC convolutional codes", in Proceedings of the 2007 IEEE Intern. Symposium on Information Theory, Nice, France, June 2007.

[9] G. Solomon and H. C. A. van Tilborg, “ A connection between block and convolutional codes”, SIAM J. Appl. Math., vol. 37, pp. 358-369, 1979.

[10] J. H. Ma, and J. K. Wolf, "On tail-biting convolutional codes”, IEEE Trans. Commun., vol. 34, pp. 104-111, 1986.

[11] R. Johannesson and K. Sh. Zigangirov, Fundamentals of Convolutional Coding, IEEE Press, 1999.

[12] R. M. Tanner, D. Sridhara, A. Sridharan, T. E. Fuja, and D. J. Costello, Jr., "LDPC block and convolutional codes based on circulant matrices”, IEEE Trans. on Inform. Theory, vol. IT-50, no. 12, pp. 2966-2984, Dec. 2004.

[13] K. Engdahl and K. Sh. Zigangirov, "On the theory of low-density convolutional codes I", Problems of Information Transmission (Problemy Peredachi Informazii), vol. 35, no. 4, pp. 295-310, Oct.-Dec. 1999.

[14] A. E. Pusane, A. Jimenez-Felström, A. Sridharan, M. Lentmaier, K. Sh. Zigangirov, and D. J. Costello, Jr., "Implementation aspects of LDPC convolutional codes", IEEE Trans. Commun. (accepted for publication), 2007.

[15] A. Sridharan, M. Lentmaier, D. V. Truhachev, D. J. Costello, Jr., and K. Sh. Zigangirov, “On the minimum distance of lowdensily parity-check codes with parity-check matrices constructed from permutation matrices", Problems of Information Transmission (Problemy Peredachi Informazii), vol. 41, no. 1, pp. 39-52, Jan.-March 2005.

[16] R. G. Gallager, Information Theory and Reliable Communication. John Wiley, New York, 1968.

[17] C. Berrou and A. Glavieux, "Near Optimum Error Correcting Coding and Decoding: Turbo-Codes", IEEE Transactions on Communications, pp. 1261-1271, vol. 44, Oct. 1996. 